\title{
Sedimentation acceleration of remanent iron oxide by magnetic flocculation
}

\author{
Mathias Stolarski ${ }^{a, *}$, Christian Eichholz ${ }^{\text {a, }}$, \\ Benjamin Fuchs ${ }^{b, 2}$, Hermann Nirschl ${ }^{a, *}$ \\ ${ }^{a}$ Institute of Mechanical Process Engineering and Mechanics (MVM), \\ University of Karlsruhe, 76131 Karlsruhe, Germany \\ ${ }^{\mathrm{b}}$ DuPont, Experimental Station, Building E304 Wilmington, DE 19880, USA
}

Received 14 June 2006; accepted 17 January 2007

\begin{abstract}
Sedimentation based processes are widely used in industry to separate particles from a liquid phase. Since the advent of the "Nanoworld" the demand for effective separation technologies has rapidly risen, calling for the development of new separation concepts, one of which lies in hybrid separation using the superposition of a magnetic field for magnetic particles. Possible product portfolio of such separation consists of pigment production, nanomagnetics production for electronics and bio separation. A promising step in that direction is magnetic field enhanced cake filtration, which has by now progressed from batch to continuous operation.

In sedimentation processes in a mass force field the settling behaviour of particles strongly depends on physico-chemical properties, concentration and size distribution of the particles. By adjusting the $\mathrm{pH}$, the interparticle forces, in particular the electrostatic repulsion, can be manipulated. For remanent magnetic particles such as magnetite, pre-treatment in a magnetic field could lead to a change of interparticle interactions. By magnetizing the particles apart from van der Waals attraction and electrostatic repulsion, an additional potential is induced, the magnetic attraction, which could easily dominate the other potentials and result in agglomeration in the primary minimum. By sedimentation analysis, a wide spectrum of parameters like $\mathrm{pH}$, magnetic field strength and concentration have been investigated. The results show a strong increase of sedimentation velocity by magnetic flocculation of the raw suspension. This leads to a rise in throughput due to the acceleration of sedimentation kinetics by imparting a non-chemical interaction to the physico-chemical properties in the feed stream of the separation apparatus.
\end{abstract}

(C) 2007 Chinese Society of Particuology and Institute of Process Engineering, Chinese Academy of Sciences. Published by Elsevier B.V. All rights reserved.

Keywords: Magnetic flocculation; Field enhanced separation; Cake filtration; Magnetic structuring; Magnetic separation

\section{Introduction}

Sedimentation based separation processes are broadly used in modern production. For coarse particles gravity sedimentation is used as exemplified by the clarifier, while for fine particles sedimentation could be enhanced by supplementing gravity with a centrifugal field. As we are entering the Nanoworld, the demand for separating smaller and smaller particles increases, for which conventional separation equipment has reached its limits.

\footnotetext{
* Corresponding authors. Tel.: +49 721608 2427; fax: +49 7216082403.

E-mail addresses: mathias.stolarski@mvm.uni-karlsruhe.de (M. Stolarski), hermann.nirschl@mvm.uka.de (H. Nirschl).

1 Tel.: +49 721608 2427; fax: +49 7216082403 .

2 Tel.: +1 302695 2146; fax: +1 3026953501 .
}

The cause for that usually lies in the forces acting on the suspended particles. For particles of sufficiently small size, their residence time may well be too short for adequate separation from the liquid stream. This physical limitation is commonly overcome by flocculation, that is, by adding chemical coagulants that affect the interparticle interactions, e.g., inorganic electrolytes, organic polymers and synthetic polyelectrolytes with either anionic or cationic functional groups. These electrolytes directly influence the repulsive and attractive interactions between approaching particles by influencing the charge distribution in the electric double layer.

For remanently magnetic materials, however, another option for manipulating interparticle interactions is possible, that is, premagnetization of the particles which would lead to very strong attractive forces between particles, causing strong flocculation without the injection of chemical additives, which 


\begin{tabular}{|c|c|}
\hline \multicolumn{2}{|c|}{ Nomenclature } \\
\hline$a$ & distance $(\mathrm{m})$ \\
\hline$A$ & Hamaker constant $(\mathrm{V} \times \mathrm{C})$ \\
\hline$c_{\mathrm{F}}$ & floc concentration (vol\%) \\
\hline$c_{\mathrm{V}}$ & particle volume concentration (vol\%) \\
\hline & floc size $(m)$ \\
\hline \multicolumn{2}{|c|}{$[\mathrm{d} p / \mathrm{d} t]_{t}$ slope of tangent at evaluation point $(\mathrm{Pa} / \mathrm{s})$} \\
\hline$g$ & earth gravity $\left(\mathrm{m} / \mathrm{s}^{2}\right)$ \\
\hline$I$ & ionic strength \\
\hline$k$ & Boltzmann constant $(\mathrm{J} / \mathrm{K})$ \\
\hline$k$ & floc building number, 1 \\
\hline$M$ & magnetization $\left(\mathrm{A} \mathrm{m}^{2} / \mathrm{kg}\right)$ \\
\hline$p_{0}$ & initial differential pressure $(\mathrm{Pa})$ \\
\hline$p_{1}$ & differential pressure at evaluation point $(\mathrm{Pa})$ \\
\hline$p_{\mathrm{Q} 1}$ & initial differential pressure at evaluation point $(\mathrm{Pa})$ \\
\hline$q$ & charge $(\mathrm{C})$ \\
\hline$Q_{3}$ & cumulative volumetric distribution, 1 \\
\hline$s$ & relative distance, $s=2(a+x) / x, 1$ \\
\hline$t_{1}$ & time at evaluation point $(\mathrm{s})$ \\
\hline$T$ & temperature $(\mathrm{K})$ \\
\hline$u_{\mathrm{fl}, \mathrm{St}}$ & Stokes single floc settling velocity $(\mathrm{m} / \mathrm{s})$ \\
\hline$u_{\mathrm{S}}$ & Richardson-Zaki settling velocity $(\mathrm{m} / \mathrm{s})$ \\
\hline$u_{\mathrm{St}}$ & Stokes settling velocity $(\mathrm{m} / \mathrm{s})$ \\
\hline$V_{\mathrm{el}}$ & electrostatic potential $(\mathrm{V} \times \mathrm{C})$ \\
\hline$V_{\text {ges }}$ & total potential $(\mathrm{V} \times \mathrm{C})$ \\
\hline$V_{\mathrm{mag}}$ & magnetic potential $(\mathrm{V} \times \mathrm{C})$ \\
\hline$V_{\mathrm{vdW}}$ & van der Waals potential $(\mathrm{V} \times \mathrm{C})$ \\
\hline$x$ & particle size $(\mathrm{m})$ \\
\hline$z$ & valency of ion, 1 \\
\hline \multicolumn{2}{|c|}{ Greek symbols } \\
\hline$\varepsilon_{\mathrm{r}}$ & dielectric number, 1 \\
\hline$\gamma$ & substitution parameter, 1 \\
\hline$\eta$ & viscosity (Pa s) \\
\hline$\kappa$ & Debye-Hückel distance (m) \\
\hline$\rho_{\mathrm{F}}$ & density of floc $\left(\mathrm{kg} / \mathrm{m}^{3}\right)$ \\
\hline$\rho_{1}$ & density of liquid $\left(\mathrm{kg} / \mathrm{m}^{3}\right)$ \\
\hline$\rho_{\mathrm{S}}$ & density of solids $\left(\mathrm{kg} / \mathrm{m}^{3}\right)$ \\
\hline$\zeta$ & zeta potential $(\mathrm{V})$ \\
\hline \multicolumn{2}{|c|}{ Constants } \\
\hline$e_{0}$ & charge of electron, $1.6 \times 10^{-19} \mathrm{C}$ \\
\hline$k$ & Boltzmann constant, $1.38 \times 10^{-23} \mathrm{~J} / \mathrm{K}$ \\
\hline$\varepsilon_{0}$ & $\begin{array}{l}\text { dielectric constant in vacuum, } \\
1.6 \times 10^{-19} \mathrm{~A} \mathrm{~s} /(\mathrm{V} \mathrm{m})\end{array}$ \\
\hline$\mu_{0}$ & $\begin{array}{l}\text { magnetic vacuum permeability, } \\
1.257 \times 10^{-6} \mathrm{~V} \mathrm{~s} /(\mathrm{A} \mathrm{m})\end{array}$ \\
\hline
\end{tabular}

might seriously affect product quality or downstream processing.

The structure of the floc strongly depends on interparticle interaction, the collision frequency and effectiveness as well as possible shear stress to the floc. Interparticle attractive forces are the necessary condition of flocculation. While increasing colli- sion frequency and effectiveness increases the floc size, shear stress results in the opposite.

\section{Theory}

Forces between particles in a liquid phase are usually described by the classical DLVO-theory (Derjaguin \& Landau, 1941; Verwey \& Overbeek, 1948), in which the particulate system is reduced to that of two approaching particles, which experience attractive as well as repulsive forces. These forces are electrostatic repulsion, van der Waals attraction and born repulsion.

Depending on the actual particle system special potentials might occur. The total potential is calculated as follows:

$V_{\text {ges }}=V_{\mathrm{vdW}}+V_{\mathrm{el}}$,

in which the van der Waals potential $V_{\mathrm{vdW}}$ and the electrostatic potential $V_{\mathrm{el}}$ are calculated as follows in Eqs. (2) and (3):

$V_{\mathrm{vdW}}=-\frac{A}{6}\left(\frac{2}{s^{2}-4}+\frac{2}{s^{2}}+\ln \frac{s^{2}-4}{s^{2}}\right)$,

$V_{\mathrm{el}}=\frac{x \varepsilon_{0} \varepsilon_{\mathrm{r}}}{z^{2}} \frac{4 k^{2} T^{2}}{e_{0}^{2}} \gamma^{2} \exp (-\kappa a)$,

With $\gamma=\left(\exp \left(\frac{I}{2}\right)-1\right)\left(\exp \left(\frac{I}{2}\right)+1\right)$,

and $I=\frac{z e_{0} \varsigma}{k T}$, as the ionic strength.

In the case of magnetized particle suspensions an interparticle magnetic potential occurs and the classical DLVO-theory needs to be modified (Chin, Yiacoumi, \& Tsouris, 2001; Chin, Yiacoumi, Tsouris, Relle, \& Grant, 2000; Svoboda, 1982a,b; Svoboda \& Zofka, 1983) as shown in Eq. (7) by rewriting Eq. (1) with $V_{\mathrm{mag}}$, the magnetic potential between two particles in Eq. (6).

$V_{\mathrm{mag}}=\frac{\pi M^{2} \mu_{0} x^{3}}{144 a^{3}}$

$V_{\mathrm{ges}}=V_{\mathrm{vdW}}+V_{\mathrm{el}}+V_{\mathrm{mag}}$.

The result for the modified DLVO-calculation for several values of magnetization is shown in Fig. 1, using as input values of the calculation data from experiments to be described later in this paper. As can be seen in Fig. 1a for non-magnetized particles, there is a strong repulsive interaction to overcome the van der Waals force before agglomeration could take place. With increasing magnetization, as shown in Fig. 1b-d, the attractive magnetic forces dominate interparticle interaction leading eventually to the disappearance of the repulsive potential.

This strong and far-reaching attraction leads to aggregation of the particles. To determine the influence of such interparticle interaction on flocculation, the sedimentation behaviour was investigated by determining the sedimentation velocity distribution in a centrifugal field (Bickert, 1997).

For highly dilute particle slurries at small Reynolds numbers in laminar flow, with small hindrance among neighbouring par- 

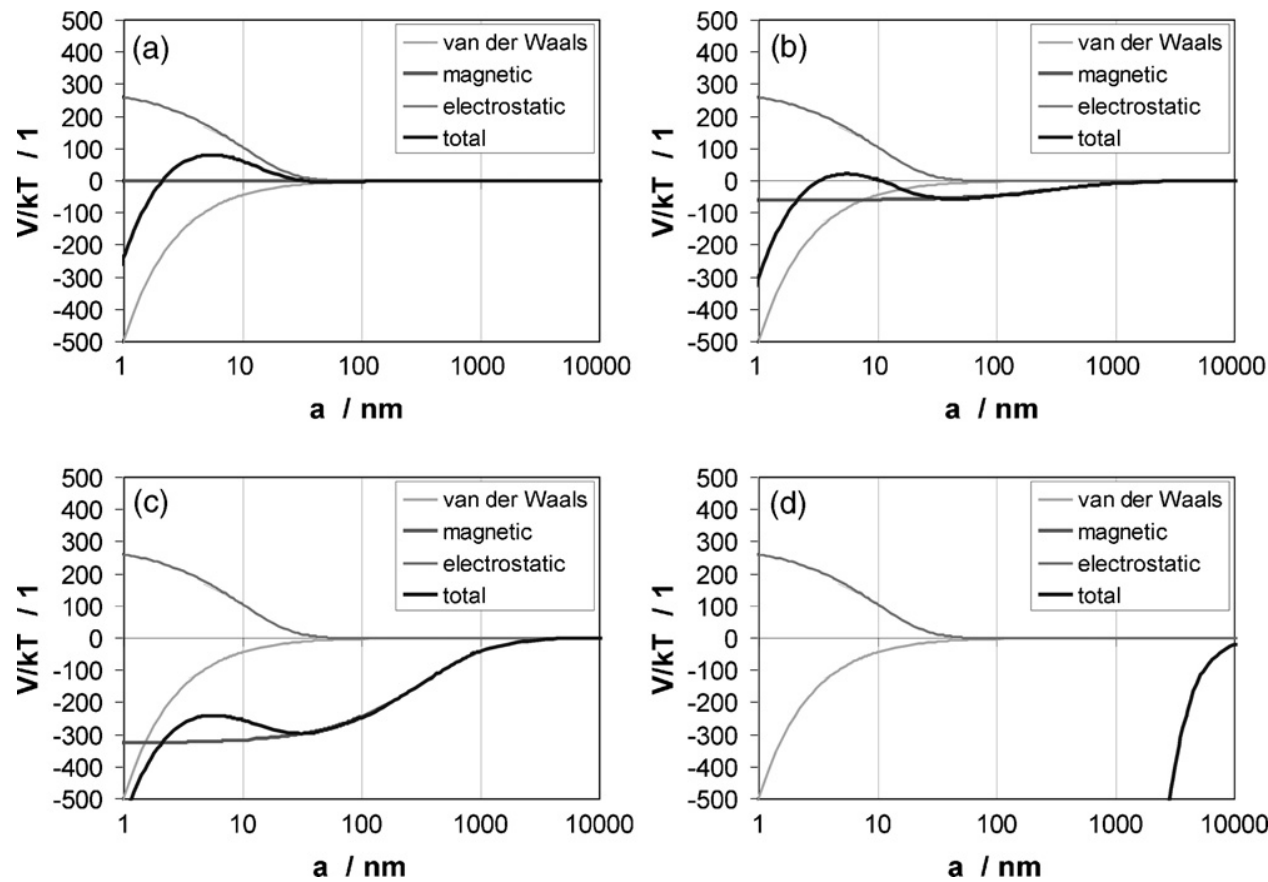

Fig. 1. DLVO potentials for different magnetizations. (a) $M=0 \mathrm{~A} \mathrm{~m}^{2} / \mathrm{kg}$, (b) $M=0.6 \mathrm{~A} \mathrm{~m}^{2} / \mathrm{kg}$, (c) $M=1.4 \mathrm{~A} \mathrm{~m}{ }^{2} / \mathrm{kg}$ and (d) $M=13 \mathrm{~A} \mathrm{~m}{ }^{2} / \mathrm{kg}$.

ticles, Stokes Law is applicable and the sedimentation velocity of single particles can be calculated as follows:

$u_{\mathrm{St}}=\frac{\left(\rho_{\mathrm{S}}-\rho_{1}\right) x^{2} g}{18 \eta}$.

Mutual hindrance among highly concentrated particles in sedimentation leads to velocities lower than those calculated by Stokes Law, which could be differentiated by the three regimes of Fig. 2: "swarm sedimentation", "zone sedimentation" and "compression" as particle concentration increases. Richardson and Zaki (1954) proposed a correction of the sedimentation velocity applicable to zone sedimentation in terms of the particle volume fraction $c_{\mathrm{v}}$ as follows:

$u_{\mathrm{S}}=u_{\mathrm{St}}\left(1-c_{\mathrm{V}}\right)^{4.65}$.

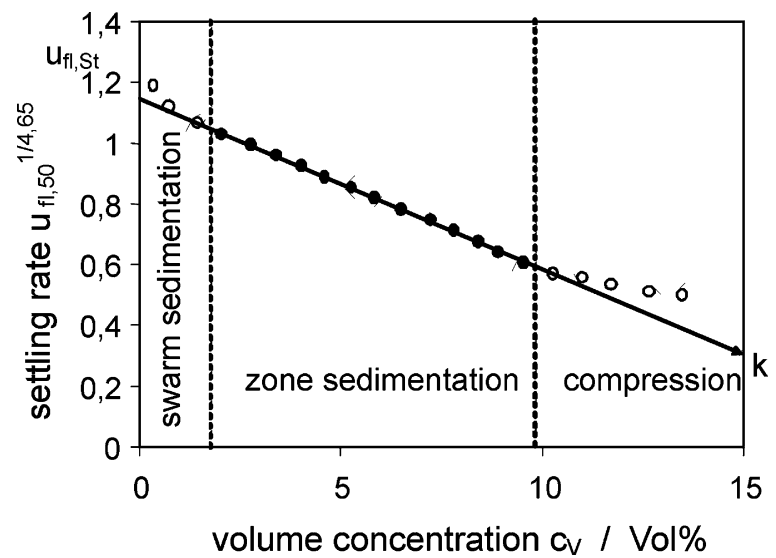

Fig. 2. Freidinger's chart for extrapolation to single floc settling speed and floc building number.
In their approach Richardson and Zaki considered the particles more as discrete than as flocculated. The sedimentation behaviour of the floc was treated by Scott (1968) in terms of the following correction:

$u_{\mathrm{S}}=u_{\mathrm{fl}, \mathrm{St}}\left(1-c_{\mathrm{F}}\right)^{4.65}$,

$c_{\mathrm{F}}=k c_{\mathrm{V}}$,

where the particle volume concentration is substituted by the volume concentration of the floc, assuming the floc to be nonporous and without inner liquid, that is, the floc concentration was considered to be equal to the product of a flocculation number $k$ and the actual solids volume concentration $c_{\mathrm{v}}$ as shown in Eq. (11).

From the above, Freidinger (1985) recommended the charting of the relative sedimentation velocity raised to the power of 1/4.65 versus the volume concentration, as shown in Fig. 2. Such correlation is linear in the region of zone sedimentation.

When extrapolating the linear zone sedimentation region towards a concentration of 0 and towards higher concentrations, the intersection with the $y$-axis is the single floc settling rate $u_{\mathrm{fl}, \mathrm{St}}$ to the $1 / 4.65$ power and the intersection with the $x$-axis is the flocculation number $k$. From those two characteristic values, $u_{\mathrm{fl}, \mathrm{St}}$ and $k$ which may be obtained by fitting experiment data based on Eqs. (10) and (11), the floc density and floc size can be calculated:

$\rho_{\mathrm{F}}=\frac{\rho_{\mathrm{s}}-\rho_{\mathrm{l}}}{k}+\rho_{\mathrm{l}}$,

$d_{\mathrm{F}}=\sqrt{\frac{18 \eta u_{\mathrm{fl}, \mathrm{St}}}{\left(\rho_{\mathrm{F}}-\rho_{\mathrm{l}}\right) g}}$. 
Table 1

Properties of Bayoxide E8709 $\left(\mathrm{Fe}_{3} \mathrm{O}_{4}\right)$

\begin{tabular}{|c|c|c|c|c|}
\hline \multirow[t]{2}{*}{ Particle size, $x_{\text {predominant }}($ producer) $(\mu \mathrm{m})$} & \multirow[t]{2}{*}{ Density, $\rho\left(\mathrm{g} / \mathrm{cm}^{3}\right)$} & \multicolumn{2}{|l|}{ Magnetization } & \multirow[t]{2}{*}{ Potential, $\zeta$ (slurry) $(\mathrm{mV})$} \\
\hline & & $M_{\mathrm{S}}$ (saturation) $\left(\mathrm{A} \mathrm{m}^{2} / \mathrm{kg}\right)$ & $M_{\mathrm{r}}($ remanent $)\left(\mathrm{A} \mathrm{m}^{2} / \mathrm{kg}\right)$ & \\
\hline 0.3 & 4.98 & 90 & 13.1 & -34 \\
\hline
\end{tabular}

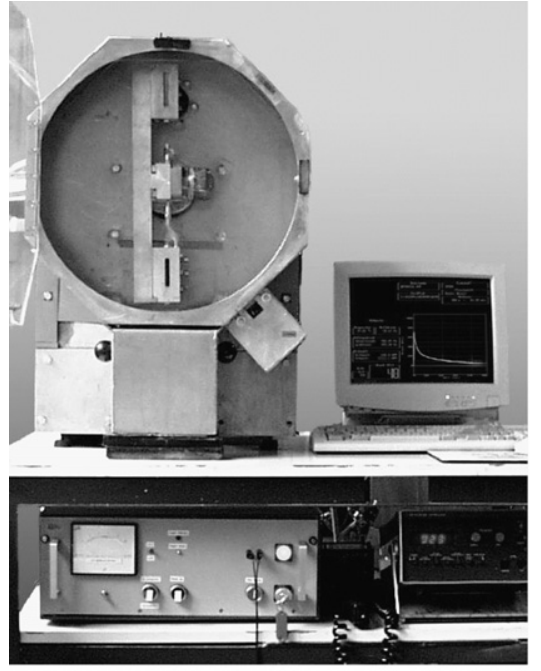

(a)

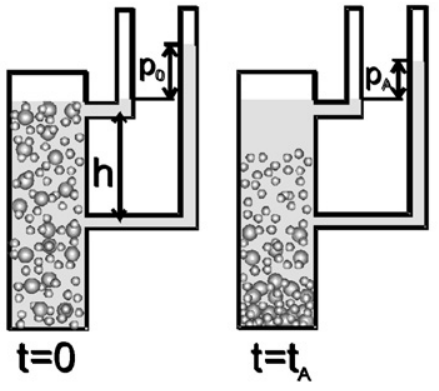

(b)

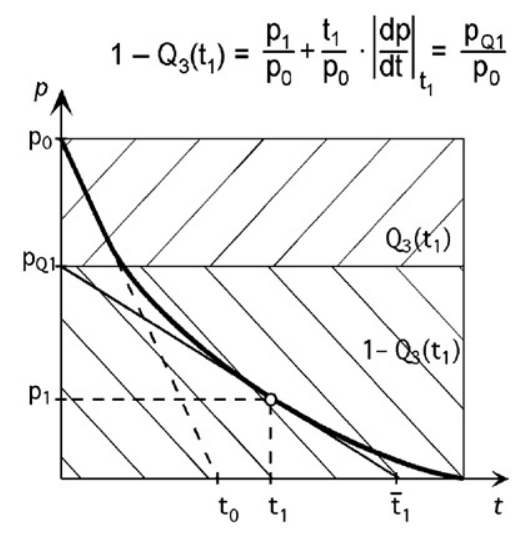

(c)

Fig. 3. Manometer principle to measure settling rate distributions via differential pressure in centrifugal field. (a) Apparatus, (b) measurement principle and (c) evaluation method.

\section{Experimental}

\subsection{Materials}

Experiments were carried out using Lanxcess Bayoxide E8709, which is a ferromagnetic iron oxide that consists mostly of $\mathrm{Fe}_{3} \mathrm{O}_{4}$ with properties shown in Table 1.

\subsection{Methods}

Sedimentation velocity distribution was measured in a centrifugal field by using the so called manometer principle (Beiser, Bickert, \& Scharfer, 2004) as shown in Fig. 3. This measurement is based on the change of mixture density due to the sedimentation of particles out of the measurement zone (Fig. 3b). The measurement zone spans two connections of a differential pressure sensor. The measured differential pressure is proportional to the concentration of the particles between the upper and the lower connections. In case of particles passing the lower connection the concentration in the measurement zone is reduced and the pressure difference decreases. From this the sedimentation velocity distribution can be calculated and analysed by a tangential method as shown in Fig. 3c. By using the given equation and a stepwise tangent method the settling rate distribution is calculated as a cumulative volumetric distribution ( $Q_{3}$-distribution) from the slope of the tangent, whereby one tangent results in one point of the distribution curve. By stepwise processing of the whole differential pressure curve a complete distribution is obtained. The experiments were performed at different concentrations and centrifugal accelerations.

\section{Results and discussion}

Fig. 4 plots the settling rate distribution of slurries of different concentrations at $\mathrm{pH} 12$ and $135 \times g$ centrifugal acceleration for non-magnetized and magnetized samples. Low-concentration slurries show higher settling rates due to lower interactions and hindrance. With increase in concentration from 3 to $5 \%$ the settling rate is reduced by a factor of 2.15 .

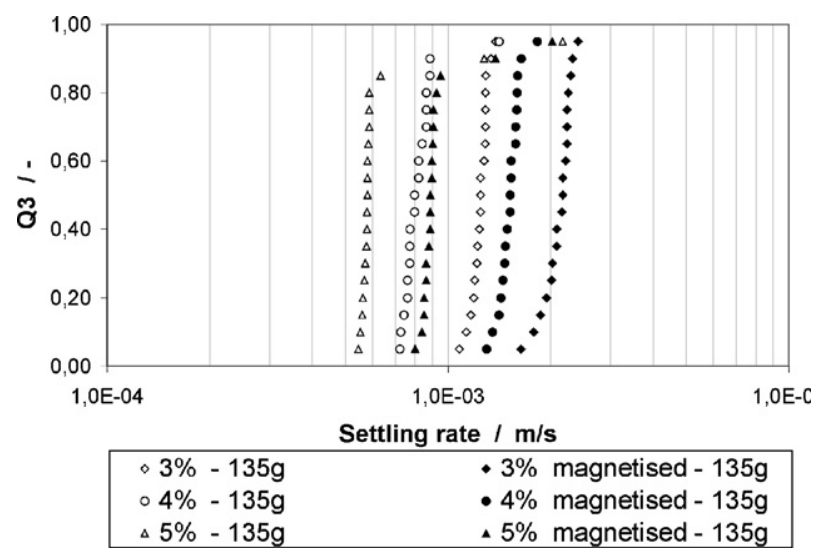

Fig. 4. Settling rate distributions for several concentrations at $135 \times g$ centrifugation acceleration. 


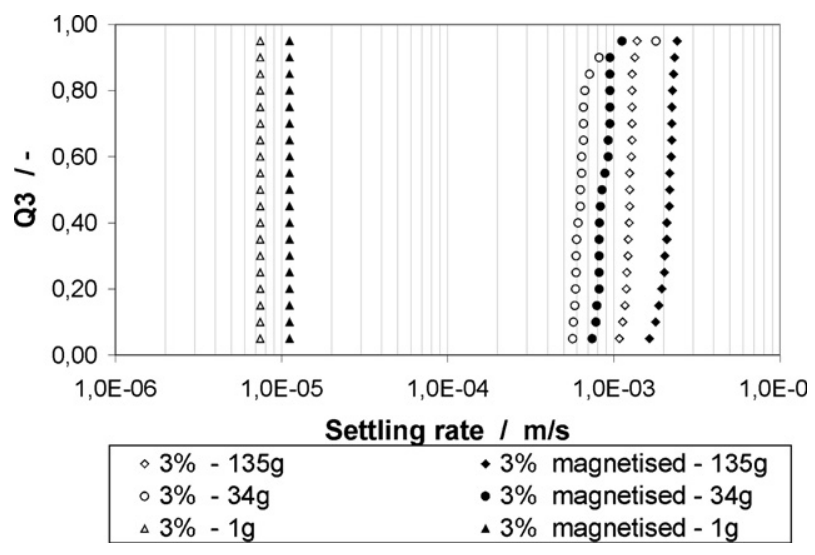

Fig. 5. Settling rate distribution for several centrifugation accelerations.

With magnetization the same rule of reduction in settling rate with increase in concentration applies, though more importantly, is the increase of settling rate due to magnetization for the same concentration. For example, in case of a volume concentration of 3\% the settling rate after magnetization is increased by a factor of 1.74 , and for $5 \%$ by 1.53 . The cause for the increased settling velocity lies in the flocculation of the particles under magnetization as shown in Fig. 1.

Fig. 5 shows the dependence of the settling rate on the magnitude of centrifugal acceleration, which varies from $1 \times g$ on the left through $34 \times g$ to $135 \times g$ on the right. The symbols indicate the experimental results. As expected the experiments for the highest centrifugal acceleration show the highest settling rates while gravity sedimentation unassisted by centrifugation shows the slowest velocity.

To directly compare the differences caused by flocculation of particles the settling rate distributions have to be related to the centrifugal acceleration. On the abscissa the settling rate is therefore divided by the experiment specific centrifugation number in order to enable comparison of the change of settling velocity due to coagulation or structure modifications as under or over proportional change in settling rate. For single particle experiments at different centrifugal velocities all experimental data would fall on one curve if the assumption of similar flow regimes is valid for the chosen set of conditions. In case the particles or flocs do not change due to shear stress at different centrifugal speeds, all experimental results will fall on the $1 \times g$ experimental result.

Fig. 6 shows the settling behaviour of the different samples independent of centrifugal acceleration. The $1 \times g$ (earth gravity) non-magnetized experiment is the slowest, followed by the $135 \times g$ non-magnetized and then the $1 \times g$ magnetized experiment. The fastest is the $34 \times g$ magnetized experiment.

Fig. 7 shows the Freidinger's chart mentioned earlier, which plots the relative settling rate raised to the $1 / 4.65$ power against volume concentration from experiments, for swarm sedimentation (particles concentrations $<2 \%$ ), constant-velocity sedimentation (2-5\% particles) and compression (5\% or higher). By linear regression and extrapolation to $0 \%$ particle concentration and to zero settling velocity the Stokes settling velocity

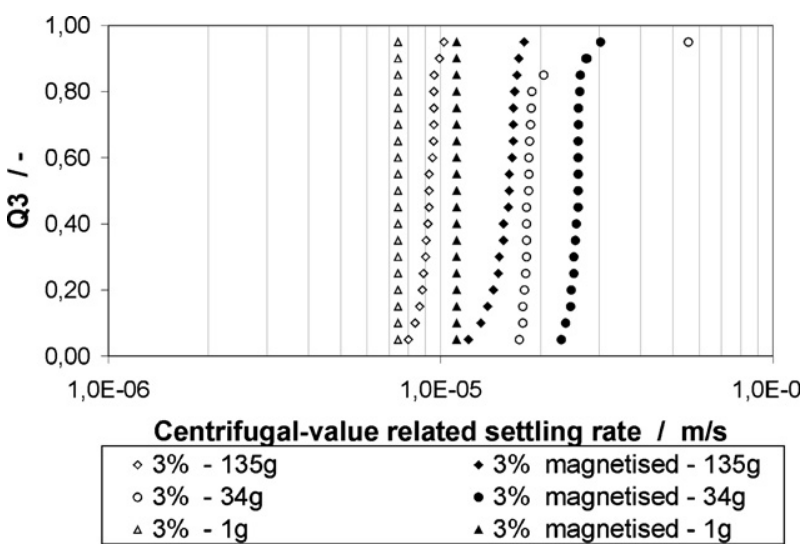

Fig. 6. Centrifugation acceleration related settling rate distributions for volume concentration of $3 \%$.

of the single floc and the so called floc building number were determined as shown in Table 2.

As stated before, mean settling velocity decreases with decreasing centrifugal acceleration. Fig. 6 shows the highest related settling velocity within the chosen centrifugal values to be measured at $34 \times g$ for the magnetized samples. Calculations show a high value of floc density and a maximum of the floc size at $34 \times g$. Here the floc size exceeds those calculated at higher as well as lower centrifugal-values by around $20 \%$. This combination of properties results in the fast settling velocity observed. The non-magnetized samples show more or less constant floc densities under centrifugal acceleration but reach a maximum density in earth gravity field. The contrary are the experiments performed with magnetized samples with a minimum density in earth gravity field, where the floc settles at lowest velocity and therefore experience a minimum shear stress. Due to the lack of shear stress flocs form freely and undisturbed which leads to branchlike agglomerate structures observed by several authors (Chantrell, Bradbury, Popplewell, \& Charles, 1982; Ozaki, Egami, Sugiyama, \& Matijevic, 1988). The developing branchlike structures are supposed to result in a loose network formation which rapidly transmits solids bulk stress. Further experiments are necessary to confirm this assumption. By applying shear to the floc a restructuring takes place that leads to a reallocation of the particles and results in a less porous floc

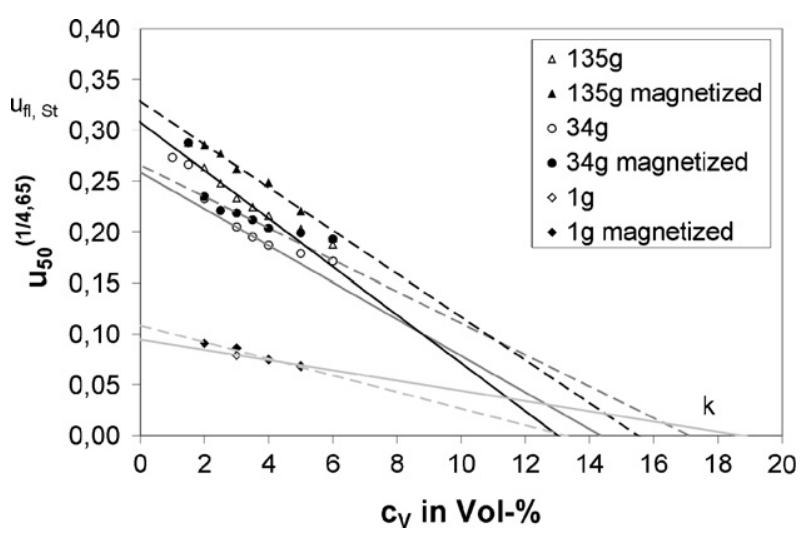

Fig. 7. Freidinger's chart for several centrifugation accelerations. 
Table 2

Calculated floc densities and floc sizes

\begin{tabular}{lllll}
\hline Experiment & Floc building number, $k$ & Single floc settling rate $(\mathrm{m} / \mathrm{s})$ & Floc density $($ Eq. $(12))\left(\mathrm{kg} / \mathrm{m}^{3}\right)$ & Floc size $(\mathrm{Eq} .(13))(\mu \mathrm{m})$ \\
\hline $135 \times g$ & 7.7 & $4.2 \times 10^{-3}$ & 1522 & 10.4 \\
$135 \times g$ magnetized & 6.5 & $5.6 \times 10^{-3}$ & 1619 & 11.1 \\
$76 \times g$ & 7.4 & $2.6 \times 10^{-3}$ & 1542 & 10.7 \\
$76 \times g$ magnetized & 5.7 & $3.0 \times 10^{-3}$ & 1701 & 10.1 \\
$34 \times g$ & 7.0 & $1.9 \times 10^{-3}$ & 1575 & 13.2 \\
$34 \times g$ magnetized & 5.8 & $2.1 \times 10^{-3}$ & 1686 & 12.9 \\
$1 \times g$ & 5.3 & $1.7 \times 10^{-5}$ & 1528 & 6.5 \\
$1 \times g$ magnetized & 7.6 & $3.2 \times 10^{-5}$ & 10.6 \\
\hline
\end{tabular}

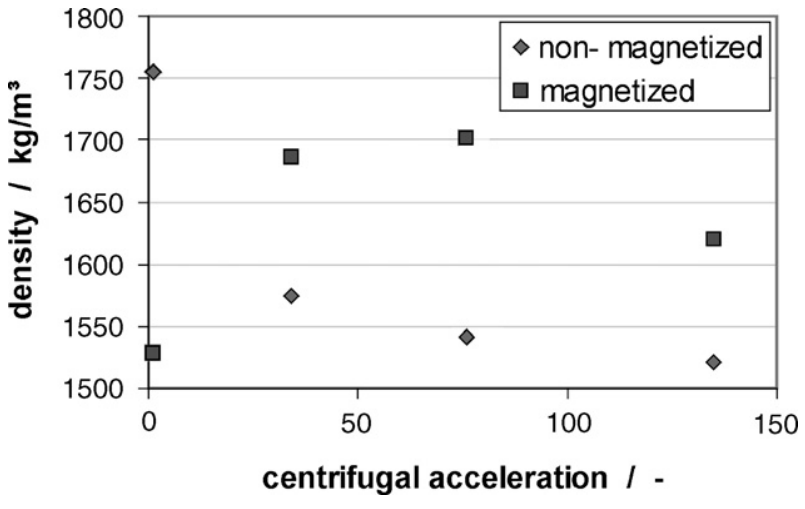

Fig. 8. Flock density as a function of centrifugal acceleration for magnetized and non-magnetized sample.

structure. Due to the inaccuracies caused by the geometric interpretation and its use in a power law the experiments have to be performed multiple and very carefully.

Flocculation is influenced by several factors. Besides the interaction potential between the particles of the slurry, other factors are the collision frequency, the collision effectiveness and the shear stress to the flocs, which is on one hand very useful as an impellent potential for collision and structural modifications, but on the other hand counter productive, because of floc destruction. At higher centrifugal acceleration the collision between particles is increased, therefore floc size increases at higher velocities in comparison to earth gravity sedimentation. The non-magnetized samples show only a $6 \mu \mathrm{m}$ minimum floc size at $1 \times g$ due to low collision frequency; this can be increased to $10 \mu \mathrm{m}$ by premagnetization as the interaction radius is increased according to Fig. 1, which directly leads to increased throughput.

Fig. 8 shows the floc density as a function of centrifugal acceleration. Due to the magnetization of the sample the density increases and stagnates at higher level in comparison to the nonmagnetized one.

\section{Conclusions}

Superposition of a magnetic field to remanent magnetic particles induces strong interparticle magnetic forces to result in flocculation which leads to the formation of compact flocs and influences the structure of the aggregates. In combination with certain flow and shear stress conditions high floc densities can be reached, that result in strong sedimentation velocity increase.
The pre-magnetization of remanent magnetic particles therefore leads to increased throughput in sedimentation-based separation processes like settling or centrifugation.

\section{Acknowledgements}

The authors owe special thanks to DuPont Engineering Research and Technology for its contribution. With the help of the Research Center Karlsruhe (FZK), Bokela GmbH, Steinert Elektromagnetbau $\mathrm{GmbH}$, the BMWi and the EU-FP6 the investigations on magnetic separation technologies can be continued and deepened. The authors also thank Lanxess for their support.

\section{References}

Beiser, M., Bickert, G., \& Scharfer, P. (2004). Comparison of sedimentation behavior and structure analysis with regard to destabilization processes in suspensions. Chemical Engineering and Technology, 27(10), 1084 1088.

Bickert, G. (1997). Sedimentation feinster suspendierter partikel im zentrifugalfeld. Institut für Mechanische Verfahrenstehnik und Mechanik (MVM), Universität Karlsruhe.

Chantrell, R. W., Bradbury, A., Popplewell, J., \& Charles, S. W. (1982). Agglomerate formation in a magnetic fluid. Journal of Applied Physics, 53(3), 2742-2744.

Chin, C., Yiacoumi, S., \& Tsouris, C. (2001). Probing DLVO-forces using interparticle magnetic forces. Langmuir, 17, 6065-6071.

Chin, C., Yiacoumi, S., Tsouris, C., Relle, S., \& Grant, S. B. (2000). Secondary-Minimum aggregation of superparamagnetic colloidal particles. Langmuir, 16, 3641-3650.

Derjaguin, B. V., \& Landau, L. D. (1941). Acta physicochim. URSS, 14, 633-662.

Freidinger, R. (1985). Untersuchungen über die ausbildung von sedimenten in kontinuierlich betriebenen absetzapparaten. Universität Stuttgart.

Ozaki, M., Egami, T., Sugiyama, N., \& Matijevic, E. (1988). Agglomeration in colloidal hematite dispersions due to weak magnetic interactions. Journal of Colloid and Interface Science, 126(1), 212-219.

Richardson, J. F., \& Zaki, W. N. (1954). Sedimentation and fluidisation. Part I. Transactions of the Institution of Chemical Engineering, 32, 35-53.

Scott, K. J. (1968). Theory of thickening: Factors affecting settling rate of solids in flocculated pulps. Transactions of the Institution of Mining and Metallurgy Section C, 77, 85-97.

Svoboda, J. (1982a). The influence of surface forces on magnetic separation. IEEE Transactions on Magnetics, 18(3), 862-865.

Svoboda, J. (1982b). Magnetic flocculation and treatment of fine weakly magnetic minerals. IEEE Transactions on Magnetics, 18(2), 796-801.

Svoboda, J., \& Zofka, J. (1983). Magnetic flocculation in secondary minimum. Journal of Colloid and Interface Science, 94, 37-44.

Verwey, E. J. W., \& Overbeek, J. T. G. (1948). Theory of stability of lyophobic colloids. Amsterdam: Elsevier. 